\title{
Prostate-specific membrane antigen expression in regeneration and repair
}

\author{
Ilyssa O Gordon ${ }^{1}$, Maria S Tretiakova ${ }^{1}$, Amy E Noffsinger ${ }^{1}$, John Hart ${ }^{1}$, Victor E Reuter ${ }^{2}$ and \\ Hikmat A Al-Ahmadie ${ }^{1}$ \\ ${ }^{1}$ Department of Pathology, University of Chicago Medical Center, Chicago, IL, USA and ${ }^{2}$ Memorial Sloan- \\ Kettering Cancer Center, New York, NY, USA
}

\begin{abstract}
Prostate-specific membrane antigen is a type II transmembrane glycoprotein, expressed in benign and neoplastic prostatic tissue as well as endothelial cells of neovasculature from a variety of tumors. The expression of prostate-specific membrane antigen in nonneoplastic neovasculature has not been well studied. Therefore, we studied nonneoplastic reparative and regenerative human tissues, as well as preneoplastic tissue, to determine the presence of prostate-specific membrane antigen-expressing neovasculature. Formalinfixed paraffin-embedded tissue from keloids, granulation tissue from heart valves and pleura, proliferative and secretory endometrium, and Barrett's mucosa with and without dysplasia were stained for the expression of prostate-specific membrane antigen (3E6). Vessels of proliferative, mid-secretory, and late secretory endometrium were consistently strongly positive for prostate-specific membrane antigen expression in all ten cases of each type (100\%). Vessels associated with granulation tissue from pleural peels and heart valves were positive in 10 of 12 cases $(83 \%)$ and 7 of 10 cases $(70 \%)$, respectively. Keloids had prostate-specific membrane antigen-expressing endothelial cells in 6 of 15 cases (40\%). Prostate-specific membrane antigen was not expressed by vessels associated with Barrett's mucosa with low-grade dysplasia (12 foci), high-grade dysplasia (24 foci), or no dysplasia (18 foci). A variety of nonneoplastic neovasculature expresses prostatespecific membrane antigen, including vessels in proliferative endometrium, granulation tissue, and some scars. This is the first study showing that prostate-specific membrane antigen is expressed in neovasculature from physiologic regenerative and reparative conditions. The folate hydrolase activity of prostate-specific membrane antigen may facilitate vasculogenesis and angiogenesis by increasing local availability of folic acid. These findings will enhance our overall understanding of blood vessel development and will enable us to better understand the effects of anti-prostate-specific membrane antigen therapies, which are already being explored in clinical trials.
\end{abstract}

Modern Pathology (2008) 21, 1421-1427; doi:10.1038/modpathol.2008.143; published online 12 September 2008

Keywords: neovasculature; vasculogenesis; folic acid; PSMA; endometrium; immunohistochemistry

Prostate-specific membrane antigen (PSMA) is a $100 \mathrm{kDa}$ type II transmembrane glycoprotein with folate hydrolase ${ }^{1}$ and neurocarboxypeptidase activity. ${ }^{2}$ Originally, PSMA expression was identified in prostate cancer cells ${ }^{3}$ and was subsequently shown to be consistently expressed in prostatic carcinoma as well as benign prostatic tissue. ${ }^{4}$ Normal epithelium of benign tissue has also been shown to express PSMA, including epithelium of the duodenum, kidney, endometrium, and breast. ${ }^{5-7}$

Correspondence: Dr HA Al-Ahmadie, MD, Department of Pathology, University of Chicago Medical Center, 5841 South Maryland Avenue, Chicago, IL 60637, USA.

E-mail: hikmat.alahmadie@uchospitals.edu

Presented at the United States and Canadian Academy of Pathology (USCAP) 97th Annual Meeting, 1-7 March 2008, Denver, CO, USA.

Received 5 May 2008; revised 8 July 2008; accepted 9 July 2008 published online 12 September 2008
PSMA has also been shown to be expressed in endothelial cells of neovasculature from a variety of tumors, including breast and renal cell carcinoma. ${ }^{8-10}$ The role of PSMA in this setting has not yet been elucidated. Although PSMA was not expressed in choroidal neovascular membranes associated with vision loss and macular degeneration in one study, ${ }^{11}$ PSMA expression has not been described in other tissues with nonneoplastic neovasculature. It is possible that the folate hydrolase activity of PSMA is important in facilitating vasculogenesis in these tissues, as well as in neoplastic neovasculature, by increasing the availability of folate, which can increase the levels of the proangiogenic molecule nitric oxide (NO) by regenerating the endothelial nitric oxide synthase (eNOS) cofactor, tetrahydrobiopterin $\left(\mathrm{BH}_{4}\right)^{12}$

In this study, we investigate the expression of PSMA in neovasculature associated with nonneoplastic reparative and regenerative human tissues. 
Our novel findings of PSMA expression in these tissues will enhance our understanding of vasculogenesis in various disease states, as well as add to our understanding of the potential impacts of antiPSMA therapies, some of which are already in clinical trials. ${ }^{13,14}$

\section{Materials and methods}

Using a protocol approved by the Institutional Review Board at the University of Chicago Medical Center, archival formalin-fixed paraffin-embedded tissue was obtained from keloids (15 cases), granulation tissue from heart valves (10 cases) and pleura (12 cases), proliferative endometrium (10 cases), and secretory endometrium (10 cases mid-secretory and 10 cases late secretory), as well as Barrett's mucosa with low- or high-grade dysplasia (12 foci and 24 foci, respectively) or no dysplasia (18 foci). Dysplastic foci in Barrett's mucosa were determined by three pathologists (IOG, AEN, and JH) who agreed on the diagnosis.

For immunohistohemical studies, $4 \mu \mathrm{m}$ sections were deparaffinized by two xylene rinses followed by two rinses with $100 \%$ ethanol. Antigen retrieval was performed by heating the slides in a microwave in a Coplin jar filled with EDTA buffer $(\mathrm{pH}$ 9.0). After rinsing briefly in $2 \times$ Tris-buffered saline (TBS) containing $0.01 \%$ Triton at $\mathrm{pH} 8$ (hereafter referred to as TBS rinses), slides were incubated for $30 \mathrm{~min}$ in $3 \%$ hydrogen peroxide in methanol to block endogenous peroxidase activity. Slides were then incubated with $0.3 \%$ bovine serum albumin in $1 \times$ TBS for $30 \mathrm{~min}$ at room temperature to reduce nonspecific background staining and then subjected to two TBS rinses. Slides were incubated for $1 \mathrm{~h}$ at room temperature with mouse anti-PSMA monoclonal antibody (clone 3E6; DAKO, Carpenteria, CA, USA; diluted 1:50). Slides were rinsed in TBS and incubated for $30 \mathrm{~min}$ with goat anti-mouse IgG conjugated to a horseradish peroxidase-labeled polymer (Envision + System; DAKO). This incubation was followed by TBS rinses, visualization with diaminobenzidine chromogen (DAKO), and then counterstained with hematoxylin. Prostate carcinoma tissue sections served as a positive control. Appropriate negative controls for the immunostaining were prepared by omitting the primary antibody step and substituting it with non-immune mouse serum. Slides were analyzed by light microscopy by two pathologists (IOG and HAA). PSMA expression in vessels was assessed, and any reactivity in vessels was considered positive. Staining intensity was scored on a scale of $0-2$, where 0 indicated no staining, 1 indicated weak staining, and 2 indicated strong staining.

\section{Results}

PSMA was expressed in the endothelial cells in 6 of $15(40 \%)$ cases of keloids (Figure 1a-c). This
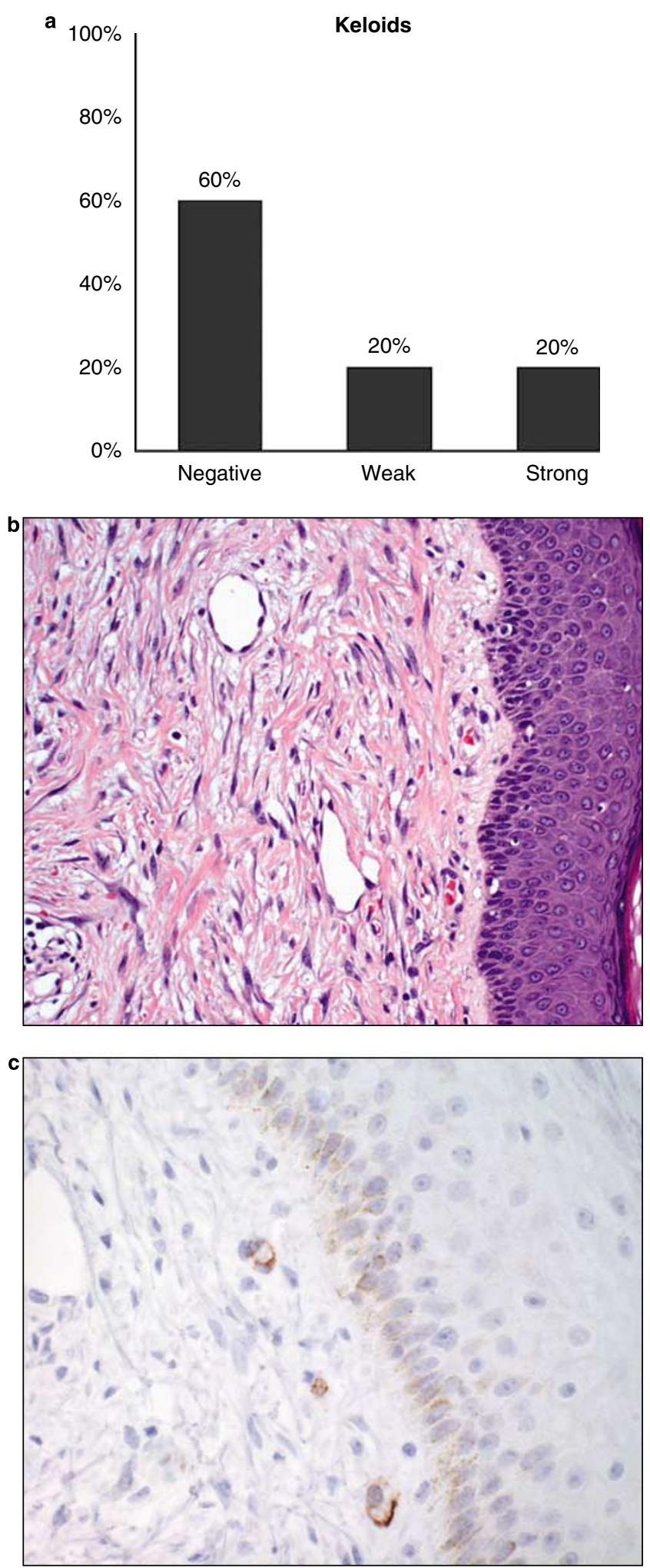

Figure 1 PSMA expression in neovasculature associated with scar tissue (a); H\&E (b) and PSMA immunohistochemistry (c).

expression was focal and limited to the papillary dermal capillaries. Half of the positive cases had weak expression, and half had strong expression. Endothelial cells associated with granulation tissue 

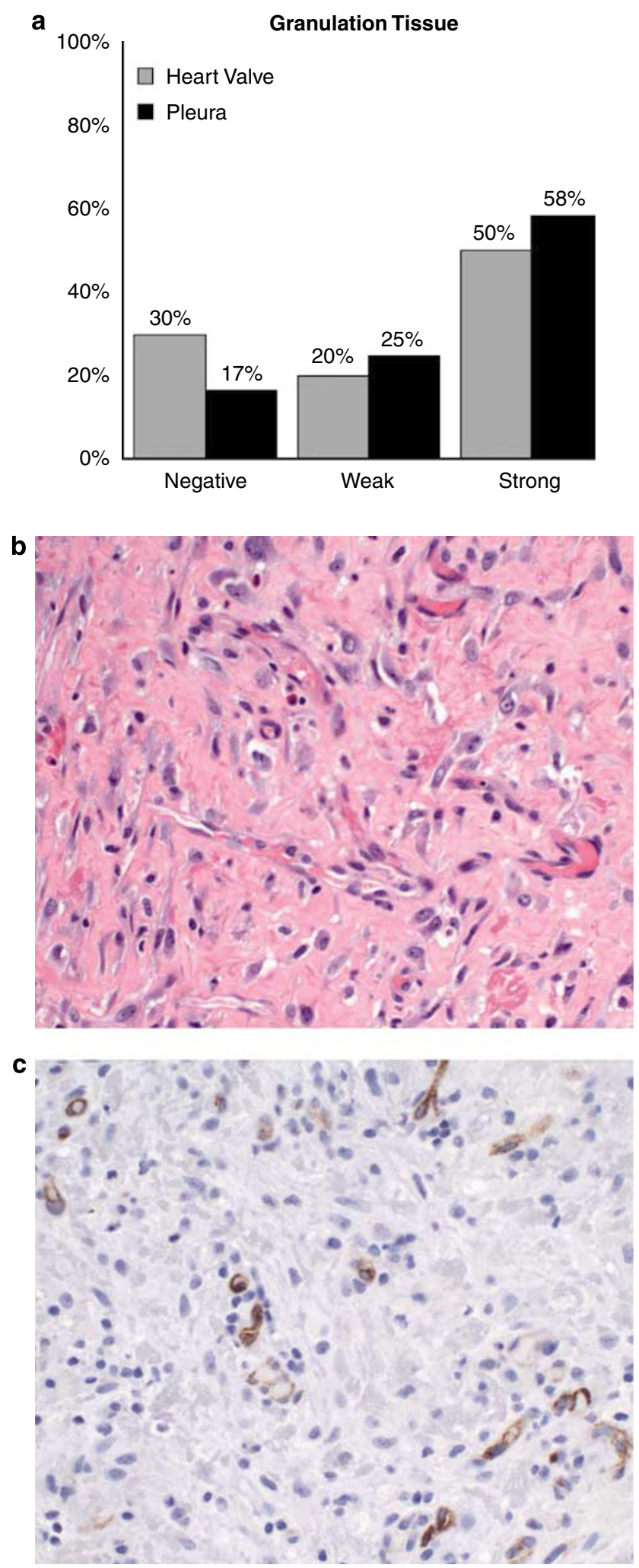

Figure 2 PSMA is expressed in granulation tissue neovasculature (a); pleural granulation tissue H\&E (b) and PSMA immunohistochemistry (c).

in heart valves and pleura expressed PSMA in 7 of $10(70 \%)$ and 10 of $12(83 \%)$ cases, respectively (Figure 2a-c). Expression was limited to capillaries and was patchy in distribution. About $30 \%$ of the positive cases had weak expression, and $70 \%$ had strong expression. All cases of mid-secretory, late secretory, and proliferative endometrium had endothelial cell PSMA expression (10 of 10 or $100 \%$ cases of each type), most having strong immunoreactivity (7 of 10 or $70 \%, 8$ of 10 or $80 \%$, and 9 of 10 or $90 \%$ of cases, respectively; Figure 3a-d). PSMA expression was present in most small vessels in a diffuse pattern. Of note, one case of midsecretory endometrium and two cases of proliferative endometrium had focal weak epithelial glandular expression of PSMA. PSMA was not expressed by vessels associated with Barrett's mucosa with or without dysplasia, including away from the lesion (Table 1).

\section{Discussion}

This is the first description of PSMA expression in neovasculature associated with nonneoplastic regenerative and reparative tissues. We found PSMA to be expressed by endothelial cells in keloids, granulation tissue from heart valves and pleura, and different phases of cycling endometrium. PSMA was not expressed by endothelium associated with Barrett's mucosa, even in the presence of associated dysplasia. In this study, the strongest and most consistent expression of PSMA in endothelial cells of neovasculature was in the endometrium, especially in the proliferative phase. The vasculature of the cycling endometrium undergoes predictable periodical changes of proliferation and regression. It expresses the proangiogenic molecules of the vascular endothelial growth factor (VEGF) family, ${ }^{15}$ and, interestingly, it has recently been shown that bone marrow-derived endothelial progenitor cells (EPCs) can contribute to the formation of endometrial neovasculature. ${ }^{16}$ Although both vasculogenesis and angiogenesis have been extensively studied in wound healing and granulation tissue, ${ }^{17}$ the exact mechanisms and pathways of neovasculature development are still unclear. Our novel finding has a variety of implications for our understanding of vasculogenesis and angiogenesis in physiologic regenerative and reparative conditions, and demonstrates that endothelial PSMA expression can occur in the absence of tumor-derived angiogenic factors.

\section{PSMA Expression in Tumor Neovasculature}

Although its expression was once thought to be exclusive to prostate epithelium, PSMA has since been shown to be expressed in neovasculature associated with a variety of tumors. Silver et $a l^{6}$ were the first to describe PSMA expression in capillary endothelium within and around renal cell carcinoma, transitional cell carcinoma of the bladder, and colonic adenocarcinoma. Subsequently, tumor neovasculature in non-small cell lung carci- 

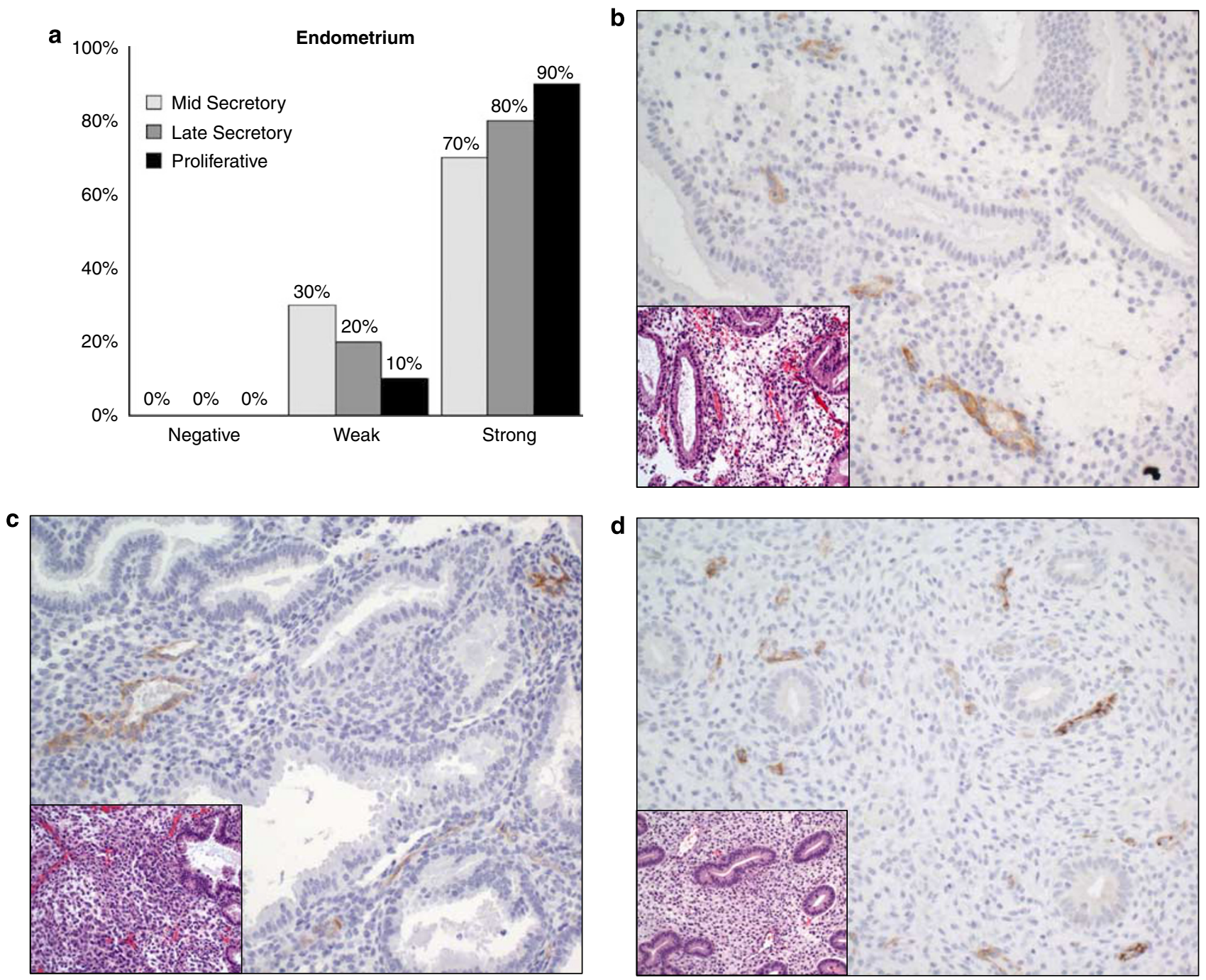

Figure 3 PSMA is strongly expressed in endometrial neovasculature (a); PSMA immunohistochemistry with H\&E inset of mid-secretory (b), late secretory (c), and proliferative (d) endometrium.

Table 1 PSMA-expressing vessels in regeneration, repair, and dysplasia

\begin{tabular}{lcccccccc}
\hline & $\begin{array}{c}\text { Proliferative } \\
\text { endometrium }\end{array}$ & $\begin{array}{c}\text { Secretory } \\
\text { endometrium }\end{array}$ & $\begin{array}{c}\text { Pleural } \\
\text { peel GT }\end{array}$ & $\begin{array}{c}\text { Heart } \\
\text { valve GT }\end{array}$ & Keloids & BM & BM-LGD & BM-HGD \\
\hline$\%$ of vessels with PSMA & 100 & 100 & 83 & 70 & 40 & 0 & 0 \\
\hline
\end{tabular}

GT: granulation tissue; BM: Barrett's mucosa; LGD: low-grade dysplasia; HGD: high-grade dysplasia; PSMA: prostate-specific membrane antigen.

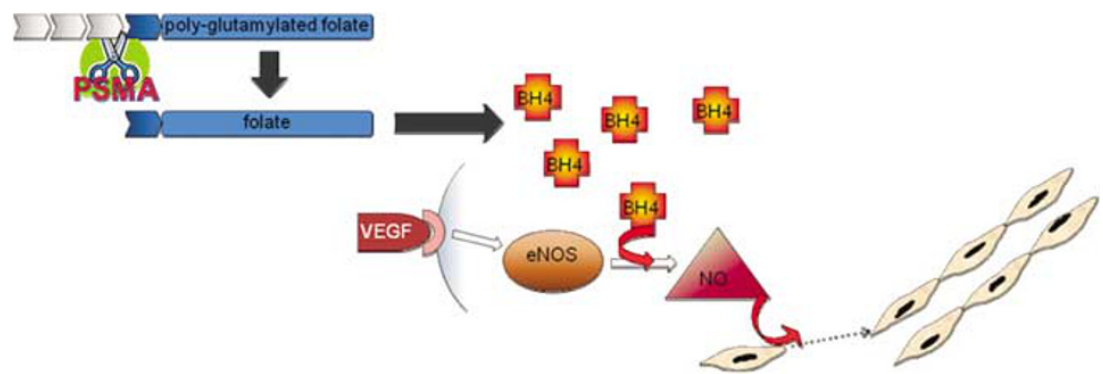

Figure 4 PSMA may facilitate vasculogenesis in reparative and regenerative tissues by increasing the availability of folate, which regenerates the eNOS cofactor $\mathrm{BH}_{4}$, permitting $\mathrm{NO}$ production and recruitment of endothelial progenitor cells. 
noma, breast carcinoma, prostate carcinoma, neuroendocrine carcinoma, pancreatic ductal carcinoma, glioblastoma multiforme, melanoma, soft tissue sarcoma, and testicular embryonal carcinoma was found to have endothelial PSMA expression in most cases. ${ }^{8,9}$ Further studies by Chang et $a l^{18}$ demonstrated PSMA mRNA in tumor endothelial cells, confirming that endothelial cells produce PSMA. Interestingly, no expression of PSMA was found in neoplastic endothelial cells in a number of vascular tumors. ${ }^{18}$ Because capillaries in adjacent normal epithelium did not express PSMA,, ${ }^{6,8,9,18}$ it was hypothesized that PSMA expression in tumorassociated neovasculature was dependent on tumor-associated angiogenic factors. ${ }^{11}$

\section{Vasculogenesis and the Role of eNOS and NO}

Our current understanding of vasculogenesis has developed rapidly over the past few years, and the characterization of EPCs, which migrate from the bone marrow in response to vasculogenic signaling molecules, is the subject of several excellent review articles. ${ }^{17,19-24}$ The role of VEGF in angiogenesis is well known, and its role in vasculogenesis has recently been elucidated (reviewed in Duda et $a l^{25}$ ). Angiogenic growth factors, including VEGF, signal through their receptors to activate eNOS, which catalyzes the breakdown of L-arginine to L-citrulline and NO (Figure 4). The importance of both eNOS and NO in EPC mobilization and vasculogenesis has recently been characterized in the setting of diabetic wound healing ${ }^{21,23}$ and in asthma vascular remodeling. ${ }^{26}$ Inhibitors of eNOS decrease VEGF-induced angiogenesis. ${ }^{27,28}$ In addition, ischemia-induced angiogenesis is reduced in eNOS knockout mice, ${ }^{29}$ and in rat models, oral folate can rescue this process in a NO-dependent manner. ${ }^{30}$

eNOS production of NO depends on the cofactor $\mathrm{BH}_{4}{ }^{31}$ The active form of folic acid, 5-methyltetrahydrofolate, restores $\mathrm{BH}_{4}$ to allow participation in eNOS reactions. ${ }^{12}$ When $\mathrm{BH}_{4}$ is not available, eNOS generates superoxide radicals, ${ }^{31}$ which impair endothelial proliferation. ${ }^{32,33}$ Folic acid and its metabolites are also superoxide radical scavengers. $^{34,35}$

\section{The Role of PSMA in Vasculogenesis}

Cellular folate gradients across cell membranes are maintained by the $\gamma$-glutamylated form, polyglutamyl folate, which accumulates intracellularly, and must be deconjugated by pteroylpoly- $\gamma$-glutamate hydrolase, also called folate hydrolase, into a monoglutamylated form to participate in metabolic reactions. ${ }^{36}$ PSMA has folate hydrolase activity. ${ }^{1}$ Therefore, our findings of PSMA expression in endothelial cells associated with neovasculature may indicate that it is functioning to increase folic acid levels at the site of neovasculature formation, so that folic acid can exert its proangiogenic effects (Figure 4). In support of this, PSMA has been reported to coimmunoprecipitate with the caveolae marker, caveolin $1,{ }^{37}$ which also coimmunoprecipitates with eNOS, ${ }^{38,39}$ suggesting the in vivo proximity of PSMA and eNOS.

Explanations for the role of PSMA in angiogenesis have been studied in the context of its expression in tumor-associated neovasculature. Conway et $a l^{40}$ found that PSMA participates in an autoregulatory loop involving $\beta 1$-integrin and p21-activated kinase 1 (PAK1) which is necessary for angiogenesis and endothelial cell invasion. Our current hypothesis complements these findings in proposing an alternative role for PSMA in neovasculature development, and specifically in vasculogenesis. It is apparent that PSMA does not play a role in all types or stages of neovasculature formation, because we did not find it to be expressed in Barrett's mucosa, with or without dysplasia, conditions known to have new vessel formation. ${ }^{41}$

\section{Implications of Current Anti-PSMA Therapies}

The strong expression of PSMA in neoplastic prostatic epithelium has led rapidly to clinical trials investigating the therapeutic use of anti-PSMA antibodies. J591 is a humanized monoclonal antibody to PSMA, which is currently being evaluated in clinical trials as a treatment modality for metastatic prostate cancer therapy. ${ }^{13,14,42}$ Both immunotherapy and targeted radiotherapy with J591 are being evaluated, and have thus far shown limited toxicity and no adverse immunologic effects. ${ }^{13,14}$ These trials were undertaken when endothelial PSMA expression was thought to be limited to neoplastic neovasculature. Our current finding of PSMA expression in nonneoplastic neovasculature, and its likely role in vasculogenesis and angiogenesis associated with reparative and regenerative conditions, is important to consider when determining the enrollment criteria for clinical trials using anti-PSMA therapies, such as regarding patients with cardiovascular disease, diabetes, and asthma.

\section{Summary}

In summary, we found PSMA expression in endothelial cells of physiologic regenerative and reparative neovasculature, which has not been described previously. This is also the first study to show endothelial PSMA expression outside of a tumor and in the absence of tumor-derived angiogenic factors. We hypothesize that in regenerative and reparative tissues, the folate hydrolase activity of PSMA increases the local availability of folic acid, thereby increasing the amount of $\mathrm{BH}_{4}$ available allowing for the VEGF-mediated production of 
NOS by eNOS, resulting in vasculogenesis and angiogenesis.

\section{Disclosure/conflict of interest}

The authors do not have any conflict of interest in the material presented.

\section{References}

1 Carter RE, Feldman AR, Coyle JT. Prostate-specific membrane antigen is a hydrolase with substrate and pharmacologic characteristics of a neuropeptidase. Proc Natl Acad Sci USA 1996;93:749-753.

2 Pinto JT, Suffoletto BP, Berzin TM, et al. Prostatespecific membrane antigen: a novel folate hydrolase in human prostatic carcinoma cells. Clin Cancer Res 1996;2:1445-1451.

3 Horoszewicz JS, Kawinski E, Murphy GP. Monoclonal antibodies to a new antigenic marker in epithelial prostatic cells and serum of prostatic cancer patients. Anticancer Res 1987;7:927-935.

4 Bostwick DG, Pacelli A, Blute M, et al. Prostate specific membrane antigen expression in prostatic intraepithelial neoplasia and adenocarcinoma: a study of 184 cases. Cancer 1998;82:2256-2261.

5 Troyer JK, Beckett ML, Wright Jr GL. Detection and characterization of the prostate-specific membrane antigen (PSMA) in tissue extracts and body fluids. Int J Cancer 1995;62:552-558.

6 Silver DA, Pellicer I, Fair WR, et al. Prostate-specific membrane antigen expression in normal and malignant human tissues. Clin Cancer Res 1997;3: 81-85.

7 Mhawech-Fauceglia P, Zhang S, Terracciano L, et al. Prostate-specific membrane antigen (PSMA) protein expression in normal and neoplastic tissues and its sensitivity and specificity in prostate adenocarcinoma: an immunohistochemical study using mutiple tumour tissue microarray technique. Histopathology 2007;50: 472-483.

8 Liu H, Moy P, Kim S, et al. Monoclonal antibodies to the extracellular domain of prostate-specific membrane antigen also react with tumor vascular endothelium. Cancer Res 1997;57:3629-3634.

9 Chang SS, Reuter VE, Heston WD, et al. Five different anti-prostate-specific membrane antigen (PSMA) antibodies confirm PSMA expression in tumor-associated neovasculature. Cancer Res 1999;59:3192-3198.

10 Al-Ahmadie HA, Olgac S, Gregor PD, et al. Expression of prostate-specific membrane antigen in renal cortical tumors. Mod Pathol 2008;21:727-732.

11 Godeiro KD, Frota AC, Antecka E, et al. Prostatespecific membrane antigen is undetectable in choroidal neovascular membrane. J Carcinog 2006;5:21.

12 Stroes ES, van Faassen EE, Yo M, et al. Folic acid reverts dysfunction of endothelial nitric oxide synthase. Circ Res 2000;86:1129-1134.

13 Nanus DM, Milowsky MI, Kostakoglu L, et al. Clinical use of monoclonal antibody HuJ591 therapy: targeting prostate specific membrane antigen. J Urol 2003;170: S84-S88; discussion S88-89.

14 Milowsky MI, Nanus DM, Kostakoglu L, et al. Vascular targeted therapy with anti-prostate-specific membrane antigen monoclonal antibody J591 in advanced solid tumors. J Clin Oncol 2007;25:540-547.

15 Mints M, Blomgren B, Falconer C, et al. Expression of the vascular endothelial growth factor (VEGF) family in human endometrial blood vessels. Scand J Clin Lab Invest 2002;62:167-175.

16 Mints M, Jansson M, Sadeghi B, et al. Endometrial endothelial cells are derived from donor stem cells in a bone marrow transplant recipient. Hum Reprod 2008;23:139-143.

17 Bauer SM, Bauer RJ, Velazquez OC. Angiogenesis, vasculogenesis, and induction of healing in chronic wounds. Vasc Endovascular Surg 2005;39: 293-306.

18 Chang SS, O'Keefe DS, Bacich DJ, et al. Prostatespecific membrane antigen is produced in tumorassociated neovasculature. Clin Cancer Res 1999;5: 2674-2681.

19 Aghi M, Chiocca EA. Contribution of bone marrowderived cells to blood vessels in ischemic tissues and tumors. Mol Ther 2005;12:994-1005.

20 Carmeliet P. Angiogenesis in health and disease. Nat Med 2003;9:653-660.

21 Brem H, Tomic-Canic M. Cellular and molecular basis of wound healing in diabetes. J Clin Invest 2007;117: 1219-1222.

22 Zammaretti P, Zisch AH. Adult 'endothelial progenitor cells'. Renewing vasculature. Int J Biochem Cell Biol 2005;37:493-503.

23 Gallagher KA, Liu ZJ, Xiao M, et al. Diabetic impairments in NO-mediated endothelial progenitor cell mobilization and homing are reversed by hyperoxia and SDF-1 alpha. J Clin Invest 2007;117: 1249-1259.

24 Caplice NM, Doyle B. Vascular progenitor cells: origin and mechanisms of mobilization, differentiation, integration, and vasculogenesis. Stem Cells Dev 2005;14: 122-139.

25 Duda DG, Fukumura D, Jain RK. Role of eNOS in neovascularization: NO for endothelial progenitor cells. Trends Mol Med 2004;10:143-145.

26 Bhandari V, Choo-Wing R, Chapoval SP, et al. Essential role of nitric oxide in VEGF-induced, asthma-like angiogenic, inflammatory, mucus, and physiologic responses in the lung. Proc Natl Acad Sci USA 2006;103:11021-11026.

27 Ziche M, Morbidelli L, Masini E, et al. Nitric oxide mediates angiogenesis in vivo and endothelial cell growth and migration in vitro promoted by substance P. J Clin Invest 1994;94:2036-2044.

28 Ziche M, Morbidelli L, Choudhuri R, et al. Nitric oxide synthase lies downstream from vascular endothelial growth factor-induced but not basic fibroblast growth factor-induced angiogenesis. J Clin Invest 1997;99: 2625-2634.

29 Murohara T, Asahara T, Silver M, et al. Nitric oxide synthase modulates angiogenesis in response to tissue ischemia. J Clin Invest 1998;101:2567-2578.

30 Sasaki K, Duan J, Murohara T, et al. Rescue of hypercholesterolemia-related impairment of angiogenesis by oral folate supplementation. J Am Coll Cardiol 2003;42:364-372.

31 Schmidt TS, Alp NJ. Mechanisms for the role of tetrahydrobiopterin in endothelial function and vascular disease. Clin Sci (Lond) 2007;113:47-63.

32 Das U. A radical approach to cancer. Med Sci Monit 2002;8:RA79-RA92. 
33 Marikovsky M, Nevo N, Vadai E, et al. Cu/Zn superoxide dismutase plays a role in angiogenesis. Int J Cancer 2002;97:34-41.

34 Verhaar MC, Stroes E, Rabelink TJ. Folates and cardiovascular disease. Arterioscler Thromb Vasc Biol 2002;22:6-13.

35 Moat SJ, Lang D, McDowell IF, et al. Folate, homocysteine, endothelial function and cardiovascular disease. J Nutr Biochem 2004;15:64-79.

36 Quinlivan EP, Hanson AD, Gregory JF. The analysis of folate and its metabolic precursors in biological samples. Anal Biochem 2006;348:163-184.

37 Anilkumar G, Barwe SP, Christiansen JJ, et al. Association of prostate-specific membrane antigen with caveolin-1 and its caveolae-dependent internalization in microvascular endothelial cells: implications for targeting to tumor vasculature. Microvasc Res 2006;72:54-61.

38 Garcia-Cardena G, Fan R, Stern DF, et al. Endothelial nitric oxide synthase is regulated by tyrosine phos- phorylation and interacts with caveolin-1. J Biol Chem 1996;271:27237-27240.

39 Goligorsky MS, Li H, Brodsky S, et al. Relationships between caveolae and eNOS: everything in proximity and the proximity of everything. Am J Physiol Renal Physiol 2002;283:F1-F10.

40 Conway RE, Petrovic N, Li Z, et al. Prostate-specific membrane antigen regulates angiogenesis by modulating integrin signal transduction. Mol Cell Biol 2006;26:5310-5324.

41 Sihvo EI, Ruohtula T, Auvinen MI, et al. Simultaneous progression of oxidative stress and angiogenesis in malignant transformation of Barrett esophagus. J Thorac Cardiovasc Surg 2003;126:1952-1957.

42 Bander NH, Trabulsi EJ, Kostakoglu L, et al. Targeting metastatic prostate cancer with radiolabeled monoclonal antibody J591 to the extracellular domain of prostate specific membrane antigen. J Urol 2003; 170:1717-1721. 